\title{
Validation du SF-36, questionnaire générique de la qualité de vie liée à la santé chez les personnes âgées au Liban
}

\author{
Nada El Osta, ${ }^{1}$ Fatme Kanso, ${ }^{2}$ Robert Saad, ${ }^{3}$ Lydia Rabbaa Khabbaz, ${ }^{4}$ Jihad Fakhouri ${ }^{1}$ et Lana El Osta ${ }^{5}$
}

${ }^{1}$ Département de Prothèse amovible, Faculté de Médecine dentaire, Université Saint-Joseph, Beyrouth (Liban) ; équipe d'accueil EA 4847, Centre de Recherche en Odontologie Clinique (CROC), Université Clermont Auvergne, Clermont-Ferrand (France); Laboratoire de Recherche Crâniofaciale, Unité de Santé Orale, Faculté de Médecine Dentaire, Université Saint-Joseph de Beyrouth, Liban. ${ }^{2}$ Faculté de Médecine, Université Saint-Joseph, Beyrouth (Liban). ${ }^{3}$ Santé publique, Université Saint-Joseph, Beyrouth (Liban). ${ }^{4}$ Laboratoire de Pharmacologie, Pharmacie clinique et Contrôle de Qualité des Médicaments, Faculté de Pharmacie, Université Saint-Joseph, Beyrouth (Liban). ${ }^{5}$ Département de Santé publique, Faculté de Médecine, Université Saint-Joseph, Beyrouth (Liban). (Correspondance à adresser à : pronada99@hotmail.com).

\section{Résumé}

Contexte : Le SF-36 est un instrument de référence pour les enquêtes de qualité de vie menées en recherche clinique et en santé publique mais aucun questionnaire n'a été validé chez les personnes âgées au Liban.

Objectif : Évaluer les propriétés psychométriques du SF-36 dans un groupe de Libanais âgés de 60 ans et plus.

Méthodes : Il s'agit d'une étude observationnelle transversale. Un échantillon de personnes âgées de 60 ans et plus a été sélectionné dans un dispensaire et dans les cliniques d'un hôpital privé et d'un hôpital gouvernemental au Liban. L'entretien personnel avait inclus des questions sur les caractéristiques sociodémographiques, la satisfaction de la santé, le score d'activité et le SF-36. Des questions ont été posées sur la présence de problèmes de santé, le nombre de médicaments. L'examen clinique comportait l'examen du poids, de la taille, ainsi que la présence d'oedème au niveau des membres inférieurs.

Résultats : Deux cent cinquante Libanais âgés de 60 ans et plus ont été inclus. Le coefficient de corrélation intraclasse variait entre 0,675 (item Q2) et 1,000 (items Q14, Q16, Q18). Le coefficient alpha de Cronbach était supérieur à 0,798. Le SF-36 était apte à discriminer les participants selon les caractéristiques sociodémographiques et la santé générale.

Conclusion : Les propriétés psychométriques du SF-36 étaient satisfaisantes sur le groupe de Libanais âgés de 60 ans et plus. Le SF-36 pourra être utilisé dans les études nationales de santé des personnes âgées.

Mots clés : Personnes âgées, Libanais, qualité de vie, santé, SF36

Citation: El Osta N; Kanso F; Saad R; Rabbaa Khabbaz L; Fakhouri1 J; El Osta L. Validation du SF-36, questionnaire générique de la qualité de vie liée à la santé chez les personnes âgées au Liban. East Mediterr Health J. 2019;25(10):706 - 714. https://doi.org/10.26719/emhj.19.041

Reçu : 03/07/18; accepté : 30/10/18

(c) Organisation mondiale de la Santé 2019. Certains droits réservés. La présente publication est disponible sous la licence Creative Commons Attribution - Pas d'utilisation commerciale - Partage dans les mêmes conditions 3.0 IGO (CC BY-NC-SA 3.0 IGO ; (https://creativecommons.org/ licenses/by-nc-sa/3.0/igo).

\section{Introduction}

L'augmentation de l'espérance de vie, l'amélioration des méthodes de soins, le changement d'attitude des personnes face à la santé ont fait émerger une nouvelle position paradigmatique dans le champ des soins de santé et de la qualité de vie. La qualité de vie est un concept abstrait et multidimensionnel relié à la satisfaction, au bien-être, à l'aisance matérielle, à l'atteinte des buts et à la volonté. Actuellement, l'évaluation de la qualité de vie occupe une place fondamentale dans la dimension de la santé et fait l'objet de très nombreuses recherches. La santé physique ne suffit pas à expliquer les différences de niveau de qualité de vie ; il est donc fondamental de prendre en compte la santé mentale et psychologique. En plus de la mortalité et de la morbidité, la qualité de vie émerge comme un outil pour évaluer les résultats des soins de santé offerts aux individus (1-3). Parmi les instruments génériques de mesure de la qualité de vie liée à la santé (QVLS), l'échelle générique 36-item Short-Form Health Survey (SF-36) [questionnaire d'évaluation de la santé en version abrégée] est applicable à tout patient quel que soit son état de santé. C'est un instrument de référence pour les enquêtes de qualité de vie menées en recherche clinique et en santé publique, très largement utilisé dans le monde (3).

Le vieillissement de la population dans le monde a favorisé l'émergence du concept de qualité de vie comme un concept fondamental au cœur des préoccupations des sciences humaines, médicales et sociales (1,4). Le Liban, comme la grande majorité des pays, est actuellement en phase de transition démographique. Malgré les progrès de la médecine, peu d'études épidémiologiques sur la perception de la santé des Libanais âgés ont été effectuées (5).

Des études de validation du SF-36 ont été réalisées chez les adultes et les enfants libanais et nous avons pu récupérer la version arabe $(6,7)$. Puisque ce questionnaire a été préalablement utilisé au Liban, son adaptation transculturelle n'est pas nécessaire selon le scénario de Guillemin (8). Toutefois, ses propriétés psychométriques doivent être évaluées chez la population gériatrique. Le but de l'étude était donc d'évaluer la validité de la version 
arabe du SF-36 auprès des personnes âgées au Liban. Notre travail contribue à l'amélioration des connaissances sur le SF-36 au niveau de la population âgée afin de pouvoir l'utiliser dans les études épidémiologiques nationales de santé. La validation d'un instrument de mesure n'est en effet jamais définitivement terminée ; son utilisation et des mesures de validation au sein de populations variées apportent des éclairages nouveaux et accroissent le nombre total de questionnaires soumis à validation.

\section{Méthodes}

\section{Population d'étude}

Il s'agit d'une étude observationnelle transversale. Le protocole a été soumis et approuvé par le comité d'éthique de l'Université Saint-Joseph de Beyrouth (USJ-2015-23). Un consentement écrit libre et éclairé a été obtenu par les participants. Les participants ont été sélectionnés à partir d'un dispensaire et des cliniques d'un hôpital privé et d'un hôpital gouvernemental. Les consultations médicales pour maladie aiguë, maladie chronique, bilan de routine et renouvellement du traitement médical constituaient le motif des visites des patients aux cliniques hospitalières et au dispensaire. Tous les sujets âgés de 60 ans et plus fréquentant chacun des trois lieux entre janvier et juin 2015 et ne présentant pas de problème cognitif ont été inclus. La version arabe du Mini-Mental State Examination (MMSE) a été utilisée pour évaluer quantitativement la fonction cognitive des sujets (9). Il s'agit d'un questionnaire de 30 items qui évalue les domaines cognitifs, y compris l'orientation temporelle, l'orientation des lieux, l'attention simple et complexe, la mémoire, les compétences linguistiques et la construction visuelle. Les scores globaux du MMSE vont de 0 à 30 ; les scores supérieurs à 24 sont considérés comme normaux (9). Cette étude était limitée aux personnes âgées ayant un score au MMSE de 24 et plus afin que les participants puissent répondre de manière adéquate au questionnaire. Il n'y avait pas de directives spécifiques pour calculer la taille de l'échantillon appropriée pour tester les performances du SF-36 chez la population âgée. Par conséquent, le nombre de 250 sujets à inclure a été fixé, en tenant compte de la taille de l'échantillon utilisé dans une étude similaire antérieure (7).

\section{Entretien oral et examen clinique}

Les participants ont été soumis à un interrogatoire et à un examen clinique menés par un seul examinateur. L'entretien personnel avait inclus des questions sur les caractéristiques socio-démographiques : âge, sexe, lieu de recrutement (dispensaire, hôpital privé, hôpital gouvernemental), niveau d'instruction, situation matrimoniale, s'il travaille actuellement ou ne travaille pas (retraités ou femmes au foyer ne travaillant pas en dehors de leur domicile pendant leur âge de travail).

Le critère d'autonomie fonctionnelle des personnes âgées a été quantifié par la version arabe du questionnaire AVQ (activités de la vie quotidienne). Celui-ci comporte une série de questions évaluant la capacité à assurer la propreté personnelle, s'habiller, passer à la toilette, assurer la continence, se déplacer et préparer un repas. Une échelle numérique étalée de 0 à 6 a été utilisée et le score final a été transformé en un pourcentage en divisant le score obtenu par 6 et en multipliant le tout par 100. Ainsi, un score compris entre 0 et 33 indique une dépendance sévère, un score compris entre 34 et 65 implique une dépendance modérée et un score de 66 et plus implique une faible dépendance ou une indépendance élevée (10). Des questions sur la satisfaction de la santé générale (satisfait, moyennement satisfait, non satisfait) ont été posées et la version arabe du questionnaire SF-36 a été soumise $(6,7)$.

Le SF-36 est issu du questionnaire de l'étude Medical Outcomes Study (MOS). C'est un questionnaire robuste et fiable destiné à obtenir une mesure générique de l'état de santé perceptuelle (3). Le SF-36 comporte 36 questions permettant d'évaluer la qualité de vie liée à la santé générale. Les 36 questions sont réparties sur huit dimensions correspondant chacune à un aspect différent de la santé. Ces dimensions sont le fonctionnement physique, la limitation des rôles en raison de problèmes physiques, la limitation des rôles résultant de problèmes émotionnels, la vitalité (énergie/fatigue), le bien-être émotionnel, le fonctionnement social, les douleurs corporelles, la perception de la santé générale et mentale.

Le score de chaque dimension du SF-36 constitue la variable dépendante de notre étude. Il s'agit de variables quantitatives discrètes, finies et dont l'échelle de mesure est ordinale. À la fin, un score pour chaque dimension du SF-36 a été calculé, variant de 0 à 100. Un score bas reflète une perception d'une mauvaise santé, une perte de fonction, une présence de douleur. Un score élevé reflète une perception d'une bonne santé, une absence de déficit fonctionnel et de douleur $(3,6,7)$.

L'état de santé des participants a été évalué en se référant au dossier médical des patients après l'accord du participant et de son médecin traitant. La présence ou non de problèmes de santé générale ainsi que le nombre et la nature des maladies, la sévérité de la maladie, le nombre de médicaments pris actuellement et le nombre d'hospitalisations au cours des six derniers mois ont été évalués. À noter que les maladies chroniques sévères à traitement spécifique nécessitant des soins continus sont l'insuffisance cardiaque, le diabète, la cirrhose hépatique, la broncho-pneumopathie chronique obstructive, l'insuffisance rénale chronique, l'asthme, la fibrose pulmonaire, le lymphome, les cancers, la maladie de Crohn, l'épilepsie et la polyarthrite rhumatoïde. Les maladies chroniques moins sévères sont les ulcères, le côlon irritable, l'ostéoporose, la fibromyalgie, la dysthyroïdie, la migraine, l'hypertrophie bénigne de la prostate, l'arthralgie et l'hypertension artérielle.

L'examen clinique comportait la présence d'œdème au niveau des membres inférieurs, le poids et la taille. Le poids a été mesuré sur une échelle de la balance à $50 \mathrm{~g}$ près, alors que la taille a été mesurée à l'aide d'un ruban 
à mesurer en métal. L'indice de masse corporelle (IMC) a été par la suite obtenu ; il s'agit d'un calcul simple utilisant la taille et le poids d'une personne et dont la formule est $\mathrm{IMC}=\mathrm{kg} / \mathrm{m}^{2}$, où $\mathrm{kg}$ est le poids d'une personne en kilogrammes et $\mathrm{m}^{2}$ sa taille en mètres carrés.

\section{Analyse statistique}

Le logiciel statistique SPSS (version 18, Chicago, IL) a été utilisé pour l'analyse statistique des données. Le seuil de significativité retenu correspond à $p \leq 0,05$. La normalité des distributions des scores a été évaluée par les tests de Kolmogorov-Smirnov. Les propriétés psychométriques du SF-6 ontété analysées. La reproductibilité est la capacité du questionnaire à mesurer le même phénomène avec la même précision d'une fois àl'autre. Trente participants ont rempli le questionnaire SF-36 en deux temps différents séparés de 1 à 4 semaines dans les mêmes conditions ; des coefficients de corrélation intraclasse ont été calculés avec leur intervalle de confiance à $95 \%$ (11-13). La cohérence interne étudie le lien entre les items du SF-36. Elle est mesurée par le coefficient $\alpha$ de Cronbach qui doit être supérieur à 0,6 (11-13). La validité concourante est le lien entre les scores du SF-36 et les scores d'un autre questionnaire qui mesure le (ou les) même(s) concept(s). La validité concourante a été évaluée en étudiant l'association entre les scores de chaque dimension du SF36 et les questions de satisfaction de la santé ; les tests de Kruskal-Wallis ont été menés. La validité discriminante, qui est la capacité du questionnaire à identifier des sujets qui diffèrent en termes d'état de santé, a été évaluée en étudiant l'association entre les scores du SF-36 et les caractéristiques socio-démographiques, la présence ou non de problèmes de santé, le nombre de maladies, la présence ou non d'au moins une maladie sévère et la présence ou non d'œdèmes des membres inférieurs. Les coefficients de corrélation de Spearman ont été utilisés pour étudier la corrélation entre les scores du SF-36 et les variables quantitatives. Les tests de Kruskal-Wallis et de Mann-Whitney ont été utilisés pour étudier l'association entre les scores du SF-36 et les variables qualitatives. Ces analyses univariées ont été suivies par des analyses multivariées de type régression multiple ayant pour variable dépendante chaque domaine du SF-36 et pour variables indépendantes celles présentant une valeur $p$ inférieure à 0,200 en analyse univariée. Les variables indépendantes fortement corrélées entre elles ont été exclues du modèle multivarié. Les variables finalement incluses étaient l'âge, le sexe, le niveau d'instruction, la présence ou non d'un problème de santé, le score ADL et la présence d'un œdème au niveau des membres inférieurs.

\section{Résultats}

Deux cent-cinquante Libanais âgés de 60 ans et plus (129 hommes et 121 femmes) d'âge moyen 70,69 ans (écart type [ET] 7,70) ont été inclus dans l'étude. Le taux de réponse était de 92,9\%. Les caractéristiques socio-démographiques sont présentées dans le tableau 1. Le nombre moyen de médicaments pris par jour était de 3,06 (ET 2,274), le score moyen ADL était de 5,84 (ET
$0,498)$ et l'indice moyen de masse corporelle était de 24,83 (ET 3,192). Le score moyen était le plus bas pour la dimension de limitation des rôles en raison d'un problème physique $(44,6$ [ET 47,85]) et le plus élevé pour les dimensions bien-être émotionnel (62,19 [ET 20,91]) et fonction physique $(60,28$ [ET 29,21]) (Figure 1).

\section{Reproductibilité}

Le coefficient de corrélation intraclasse variait entre 0,675 (item Q2) et 1,000 (items Q14, Q16, Q18).

\section{Cohérence interne}

Le coefficient $\alpha$ de Cronbach était de 0,926 pour la fonction physique, 0,973 pour la limitation des rôles en raison d'un problème physique, 0,978 pour la limitation des rôles en raison d'un problème émotionnel, 0,864 pour la dimension énergie/fatigue, 0,798 pour la dimension bien-être émotionnel, 0,832 pour la dimension fonctionnement social, 0,920 pour la dimension douleur corporelle et 0,851 pour la perception de la santé.

\section{Validité concourante}

Le score de chaque dimension du SF-36 était significativement plus élevé chez les participants satisfaits de leur état de santé par rapport à ceux qui étaient non satisfaits $(p<0,001)$ (Tableau 2).

\section{Validité discriminante}

Des analyses univariées suivies par des analyses multivariées ont été effectuées (Tableaux 3,4 et 5). Chez les hommes, le score de chaque dimension du SF-36 était significativement supérieur à celui des femmes, et

\begin{tabular}{lc}
\hline \multicolumn{2}{l}{ Tableau 1 Caractéristiques socio-démographiques } \\
\hline Caractéristiques & Nbre (\%) \\
\hline Lieu de recrutement & \\
\hline Clinique d'un hôpital gouvernemental & $61(24,4 \%)$ \\
Dispensaire & $127(50,8 \%)$ \\
Clinique d'un hôpital privé & $62(24,8 \%)$ \\
Niveau d'instruction & \\
\hline Illettré(e) & $70(28,0 \%)$ \\
Primaire & $76(30,4 \%)$ \\
Secondaire & $57(22,8 \%)$ \\
Baccalauréat & $27(10,8 \%)$ \\
Académique & $20(8,0 \%)$ \\
Situation matrimoniale & \\
\hline Célibataire & $6(2,4 \%)$ \\
Marié(e) & $214(85,6 \%)$ \\
Veuf(ve) & $28(11,2 \%)$ \\
Divorcé(e) & $2(0,8 \%)$ \\
Travail actuellement & \\
\hline Non (retraités, femmes au foyer) & $156(62,4 \%)$ \\
Oui & $94(37,6 \%)$ \\
\hline
\end{tabular}


Figure 1 Scores moyens au niveau de chaque dimension du SF-36

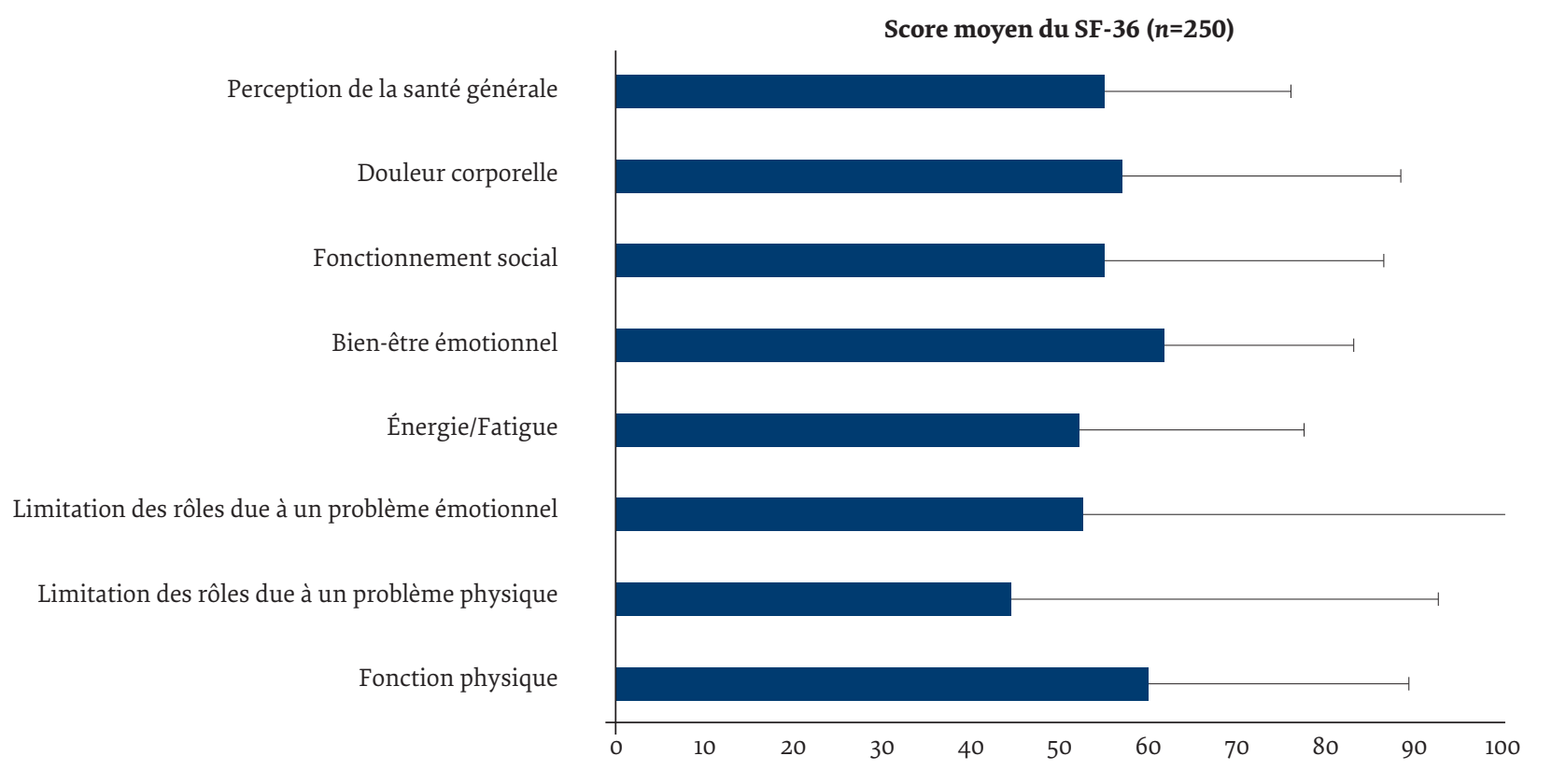

ceci pour la fonction physique $(p=0,008)$, la limitation des rôles en raison d'un problème émotionnel ( $p$ $=0,026)$, l'énergie $(p=0,002)$ et la douleur corporelle ( $p$ $=0,023$ ) (Tableaux 3 et 5). Pour les autres dimensions, la différence n'a pas été significative avec les analyses multivariées ( $p>0,05)$ (Tableau 5). D'autre part, les participants ayant un bas niveau d'instruction ont présenté un score significativement plus bas pour toutes les dimensions de SF-36 ( $p<0,05)$ (Tableaux 3 et 5 ). Les participants hospitalisés ou veufs ont présenté le score moyen significativement le plus bas, et ceci pour les huit dimensions du SF-36 ( $p<0,05)$ (Tableau 3). Toutefois, on n'a pas trouvé d'association significative entre le fait de travailler ou non et les scores du SF-36 ( $p>0,05)$ (Tableau 3). Chez les participants présentant un problème de santé, le score de chacune des huit dimensions du SF-36 était significativement inférieur à ceux n'ayant pas de problème $(p<0,05)$ (Tableaux 3 et 5). D'autre part, la sévérité des maladies présentes joue un rôle significatif, puisque les scores étaient bas en présence d'une maladie sévère (Tableau3) et d'œdème des membres inférieurs (Tableaux 3 et 5). Aussi, le score a significativement diminué chez les sujets plus âgés lorsque le nombre de médicaments pris par jour a augmenté et le degré d'autonomie a diminué (Tableaux 4 et 5). En revanche, le score du SF-36 n'a pas été corrélé avec l'IMC ( $p>0,05)$ (Tableau 4).

\section{Discussion}

L'objectif de notre étude était d'évaluer les propriétés psychométriques de la version arabe du questionnaire

\begin{tabular}{|c|c|c|c|c|}
\hline Satisfaction de la santé générale & $\begin{array}{l}\text { Non satisfait } \\
\qquad \begin{array}{c}n=34 \\
\text { (ET) }\end{array}\end{array}$ & $\begin{array}{l}\text { Moyennement } \\
\text { satisfait } \\
n=78 \\
\text { (ET) }\end{array}$ & $\begin{array}{l}\text { Satisfait } \\
\begin{array}{l}n=138 \\
\text { (ET) }\end{array}\end{array}$ & $\mathbf{P}$ \\
\hline Fonction physique & $29,56(25,36)$ & $49,49(28,37)$ & $73,95(21,31)$ & $<0,001$ \\
\hline Limitation des rôles car problème physique & $4,41(18,94)$ & $31,73(44,26)$ & $61,78(46,72)$ & $<0,001$ \\
\hline Limitation des rôles car problème émotionnel & $15,69(35,99)$ & $37,61(47,56)$ & $70,29(44,61)$ & $<0,001$ \\
\hline Énergie/Fatigue & $26,62(15,41)$ & $45,38(22,56)$ & $62,79(22,56)$ & $<0,001$ \\
\hline Bien-être émotionnel & $43,88(16,26)$ & $58,00(20,89)$ & $69,07(18,57)$ & $<0,001$ \\
\hline Fonctionnement social & $25,74(22,18)$ & $40,71(28,31)$ & $70,56(25,62)$ & $<0,001$ \\
\hline Douleur corporelle & $30,00(27,48)$ & $47,66(30,89)$ & $69,24(25,95)$ & $<0,001$ \\
\hline Perception de la santé générale & $30,00(12,49)$ & $45,13(15,08)$ & $66,85(17,02)$ & $<0,001$ \\
\hline
\end{tabular}




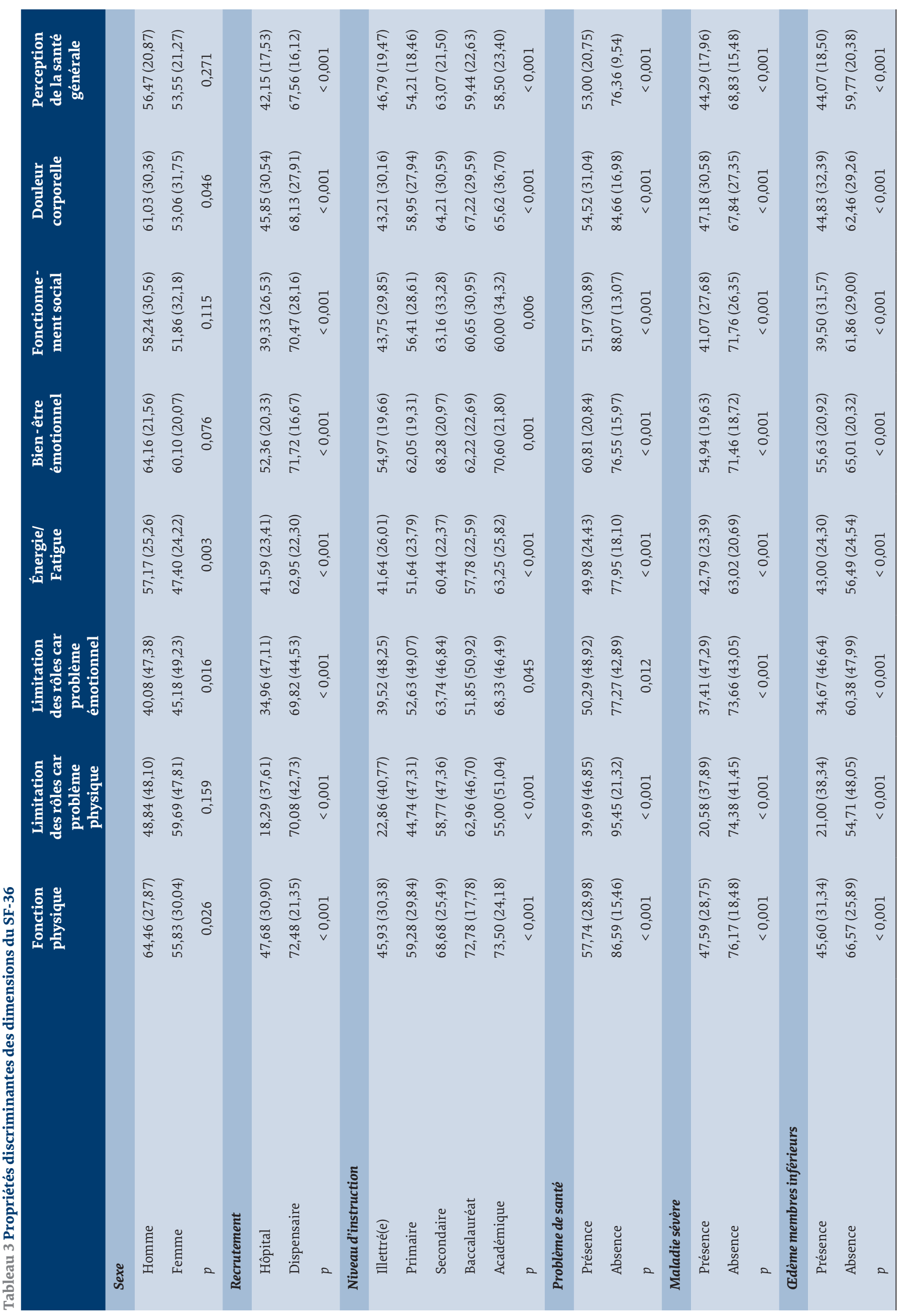




\begin{tabular}{|c|c|c|c|c|}
\hline & $\begin{array}{l}\text { Nombre de } \\
\text { médicaments }\end{array}$ & Âge & Score ADL & IMC \\
\hline \multicolumn{5}{|c|}{ Fonction physique } \\
\hline Corrélation & $-0,638$ & $-0,423$ & 0,449 & $-0,079$ \\
\hline$p$ & 0,000 & 0,000 & 0,000 & 0,212 \\
\hline \multicolumn{5}{|c|}{ Limitation des rôles car problème physique } \\
\hline Corrélation & $-0,654$ & $-0,377$ & 0,344 & $-0,071$ \\
\hline$p$ & 0,000 & 0,000 & 0,000 & 0,264 \\
\hline \multicolumn{5}{|c|}{ Limitation des rôles car problème émotionnel } \\
\hline Corrélation & $-0,398$ & $-0,155$ & 0,302 & $-0,107$ \\
\hline$p$ & 0,000 & 0,014 & 0,000 & 0,092 \\
\hline \multicolumn{5}{|c|}{ Énergie/Fatigue } \\
\hline Corrélation & $-0,565$ & $-0,273$ & 0,431 & $-0,043$ \\
\hline$p$ & 0,000 & 0,000 & 0,000 & 0,502 \\
\hline \multicolumn{5}{|c|}{ Bien-être émotionnel } \\
\hline Corrélation & $-0,448$ & $-0,159$ & 0,345 & $-0,054$ \\
\hline$p$ & 0,000 & 0,012 & 0,000 & 0,392 \\
\hline \multicolumn{5}{|c|}{ Fonctionnement social } \\
\hline Corrélation & $-0,586$ & $-0,258$ & 0,377 & $-0,065$ \\
\hline$p$ & 0,000 & 0,000 & 0,000 & 0,303 \\
\hline \multicolumn{5}{|c|}{ Douleur corporelle } \\
\hline Corrélation & $-0,498$ & $-0,253$ & 0,335 & $-0,061$ \\
\hline$p$ & 0,000 & 0,000 & 0,000 & 0,335 \\
\hline \multicolumn{5}{|c|}{ Perception de la santé générale } \\
\hline Corrélation & $-0,682$ & $-0,345$ & 0,388 & $-0,073$ \\
\hline$p$ & 0,000 & 0,000 & 0,000 & 0,247 \\
\hline
\end{tabular}

AVQ : activités de la vie quotidienne; IMC : indice de masse corporelle.

de qualité de vie liée à la santé SF-36 sur un groupe de 250 Libanais âgés de 60 ans et plus. Les résultats ont montré une bonne reproductibilité, une cohérence interne adéquate ainsi qu'une validité concourante et une validité discriminante satisfaisantes. Nos résultats rejoignent les résultats des études ayant validé le SF-36 dans leur population $(3,6,7,14,15)$. La fidélité de mesure est essentielle afin d'assurer la qualité du SF-36 (3,16). Ce concept se rapporte à la reproductibilité et à la cohérence interne du questionnaire (17). La reproductibilité de mesure du SF-36 a été évaluée chez 30 sujets et le coefficient de corrélation intraclasse était supérieur à 0,70 pour les huit dimensions, indiquant une excellente reproductibilité. Le coefficient $\alpha$ de Cronbach était supérieur à 0,798 pour les huit dimensions du SF-36, indiquant une cohérence interne élevée $(16,17)$. La validité concourante était satisfaisante puisque la qualité de vie liée à la santé (QVLS) était plus élevée chez les participants satisfaits de leur santé (14-17).

La validité discriminante d'un questionnaire est son aptitude à identifier des sujets qui diffèrent en termes d'état de santé. Par ailleurs, le SF-36 était capable de distinguer les participants selon leur état de santé. Les participants présentant au moins une maladie sévère avaient une mauvaise QVLS, comparés à ceux qui ne présentent pas de maladie sévère et ceci, pour les huit dimensions du SF-36. Pareillement, lorsque le nombre de médicaments a augmenté, la QVLS a diminué. Aussi, le SF-36 était apte à distinguer les participants selon la présence ou non d'œdèmes des membres inférieurs et selon leur autonomie dans les activités de la vie quotidienne, et ceci paraît très logique et cohérent avec la situation dans les autres pays $(8,9,14,15)$. L'évaluation de la qualité de vie est influencée évidemment par l'état de santé objectivé par le nombre et la sévérité des conditions médicales pathologiques, le nombre de médicaments, la présence d'œdèmes des membres inférieurs qui sont le reflet d'une pathologie cardiaque, rénale, hépatique. La qualité de vie, dans sa définition multidimensionnelle, est menacée par toute condition médicale, d'autant plus qu'elle est invalidante et chronique, que ce soit par une limitation sur le plan social, physique, émotionnel et professionnel.

Une validation externe a également été effectuée avec diverses variables disponibles et a priori associées à l'état de santé, notamment des variables démographiques et socio-économiques. Nos résultats avaient montré que le SF-36 était capable de distinguer les patients selon leurs 


\begin{tabular}{|c|c|c|c|c|c|c|}
\hline & \multicolumn{2}{|c|}{$\begin{array}{l}\text { Coefficients non } \\
\text { standardisés }\end{array}$} & \multirow{2}{*}{$\begin{array}{c}\text { Coefficients } \\
\text { standardisés } \\
\beta\end{array}$} & \multirow[t]{2}{*}{$t$} & \multirow[t]{2}{*}{$\mathbf{P}$} & \multirow[t]{2}{*}{$\begin{array}{c}\text { Corrélation } \\
\text { partielle }\end{array}$} \\
\hline & B & $\begin{array}{l}\text { Erreur } \\
\text { standard }\end{array}$ & & & & \\
\hline \multicolumn{7}{|l|}{ Fonction physique } \\
\hline Âge & $-0,905$ & 0,239 & $-0,238$ & $-3,791$ & 0,000 & $-0,236$ \\
\hline Sexe & $-7,858$ & 2,942 & $-0,135$ & $-2,671$ & 0,008 & $-0,169$ \\
\hline Niveau d'instruction & 5,119 & 1,245 & 0,215 & 4,111 & 0,000 & 0,255 \\
\hline Problème de santé & 15,109 & 5,316 & 0,147 & 2,842 & 0,005 & 0,179 \\
\hline Edème membre & 10,092 & 3,349 & 0,159 & 3,013 & 0,003 & 0,190 \\
\hline AVQ & 20,309 & 2,993 & 0,346 & 6,784 & 0,000 & 0,399 \\
\hline \multicolumn{7}{|c|}{ Limitation des rôles car problème physique } \\
\hline Âge & $-1,280$ & 0,425 & $-0,206$ & $-3,011$ & 0,003 & $-0,190$ \\
\hline Sexe & $-7,531$ & 5,243 & $-0,079$ & $-1,436$ & 0,152 & $-0,092$ \\
\hline Niveau d'instruction & 6,741 & 2,197 & 0,173 & 3,069 & 0,002 & 0,270 \\
\hline Problème de santé & 36,833 & 9,476 & 0,218 & 3,887 & 0,000 & 0,242 \\
\hline CEdème membre & 18,478 & 5,969 & 0,177 & 3,096 & 0,002 & 0,195 \\
\hline AVQ & 19,125 & 5,336 & 0,199 & 3,584 & 0,000 & 0,224 \\
\hline
\end{tabular}

AVQ : activités de la vie quotidienne

caractéristiques socio-démographiques. La qualité de vie liée à l'état de santé décroît chez les participants plus âgés comparés aux moins âgés. Ceci est logique puisque les seniors sont souvent des personnes vulnérables $(18,19)$. Notre étude a aussi exposé que la QVLS des hommes était supérieure à celle des femmes. Ceci est cohérent avec les résultats du Liban (6,7), de la Jordanie (18), des États-Unis (20) et du Canada (21). À l'échelle de notre pays, la femme dans le passé risquait de ne pas prendre toutes ses chances du côté professionnel et éducationnel, de ne pas satisfaire ses ambitions, de se contenter d'être une femme au foyer, en train de prendre soin de ses enfants, de son mari, des personnes âgées aux dépens de sa propre santé. Par ailleurs, sa vulnérabilité aux troubles anxio-dépressifs, prouvée dans les études épidémiologiques mondiales en psychiatrie, pourra expliquer les scores bas chez la femme âgée, surtout en ce qui concerne la santé émotionnelle. Sur le plan de la situation matrimoniale, notre étude a montré que les veuf(ve)s présentaient une mauvaise QVLS, et ceci est en accord avec les résultats d'autres études $(18,22)$. On pense que le changement matrimonial, avec le décès du partenaire, peut avoir des conséquences importantes sur l'état affectif, psychologique, sanitaire et socioéconomique de la personne, surtout s'il y avait un long accompagnement dans le chemin de la maladie et de la souffrance avant la mort (18). Ce changement influence largement le degré du sentiment de solitude chez la personne dans la mesure où la perte du conjoint peut constituer un événement qui oblige la personne à s'adapter à une nouvelle situation sociale et économique. Notre étude a montré que la QVLS décroît chez les personnes hospitalisées comparées à celles non hospitalisées. En revanche, on n'a pas trouvé d'association significative entre le fait d'être ou non à la retraite et la QVLS. D'autre part, lorsque le niveau d'instruction des participants s'améliore, leur QVLS s'améliore. Ceci est semblable au résultat obtenu en Jordanie (18) et au Maroc (22), et il est présumé, en effet, que le niveau d'instruction est étroitement lié à d'autres déterminants sociaux de la santé comme le niveau de revenu, la sécurité d'emploi et les conditions de travail. Dans cette perspective, l'éducation favorise un meilleur accès aux autres ressources sociales et économiques $(18,20,22)$. En outre, les gens acquièrent une meilleure compréhension du monde avec de meilleures habiletésetdemeilleursoutilslesmenantàdesmodesdevie plus sains.

L'étude présente certaines limites liées au manque de représentativité de l'échantillon. L'accès aux personnes âgées est difficile au Liban car il n'existe pas de système de sécurité sociale couvrant l'ensemble de la population. Ensuite, les patients ont été recrutés dans les cliniques hospitalières et un dispensaire pendant une courte période. Les résultats ne peuvent donc pas être appliqués aux personnes âgées vivant dans les communautés rurales. Les patients qui fréquentent ces structures médicales peuvent présenter des niveaux de maladie plus élevés, une meilleure couverture d'assurance maladie ou un accès plus facile aux soins médicaux. Par conséquent, les résultats de cette enquête ne peuvent pas être utilisés pour décrire la santé de la population âgée libanaise. Aussi, la taille de l'échantillon n'a pas été calculée ; cependant, les données issues de cette étude peuvent être utilisées dans des études ultérieures pour calculer le nombre nécessaire de Libanais âgés dans l'échantillon qui 
prendra en compte la prévalence du critère de jugement principal et la précision voulue de l'estimation. De cette façon, nous serons d'ailleurs en mesure de procéder aux analyses factorielles pour confirmer la construction du SF-36 en huit dimensions.

\section{Conclusion}

L'acceptabilité du SF-36 était suffisamment large, la cohérence des réponses était bonne et la fréquence de réponses manquantes était faible. Les propriétés psychométriques du SF-36 étaient satisfaisantes. Le SF-36 pourra être considéré comme un questionnaire de référence dans les recherches épidémiologiques en gériatrie au Liban. Des études devront être aussi réalisées afin de tester ses propriétés dans les études cliniques prospectives de qualité de vie liée à la santé.

Financement : aucun.

Conflits d'intérêts : aucun déclaré.

\section{Validation of the Arabic version of the SF-36, generic questionnaire of quality of life related to health among the elderly in Lebanon}

\section{Abstract}

Background: Measuring health status of elderly is essential for the prediction of their health care needs. Health research usually considers objective outcome measure; however there is a need to increase self-reported measures of health.

Aims: The aim of this study was to assess the psychometric properties of the SF36 among a group of Lebanese elderly.

Methods: It was a cross-sectional observational study. A systematic sample of elderly people aged 60 years and more was selected from dispensary, private and governmental hospitals in Lebanon. Data were collected using face-to-face interview. The first part of the questionnaire consisted of sociodemographic characteristics. The second part consisted of the Arabic version of the SF-36. The third part consisted of Activity of Daily Living (ADL) scale, presence of health problems and number of medication consumed per day.

Results: A total of 250 elderly people were included. The mean age was 70.69 \pm 7.70 years. Cronbach alphas for all SF-36 scales exceeded 0.798. The intra-Class correlation coefficient varied between 0.675 (item 2) and 0.980 (items 14, 16 and 18) indicating good reliability. The quality of life (QoL) of women was poorer than men $(p<0.001)$. It was significantly lesser when the number of health problems $(p<0.001)$ and medications $(p<0.001)$ increased, and the ADL score $(p<0.05)$ decreased. Conclusion: SF-36 is a valid and reliable instrument for measuring QoL among Lebanese elderly and could be used for monitoring the QoL of this population.

$$
\begin{aligned}
& \text { التحقق من المسح الصحي القصير المُكوَّن من بس بنداً (SF-36)، استبيان جودة الحياة الصحية العامة للمسنين في } \\
& \text { لبنان } \\
& \text { ندى الأسطا، فاطمة قانصو، روبير سعد، ليديا رباع خباز، جهاد فاخوري، لنا الأسطا } \\
& \text { الخلاصة }
\end{aligned}
$$

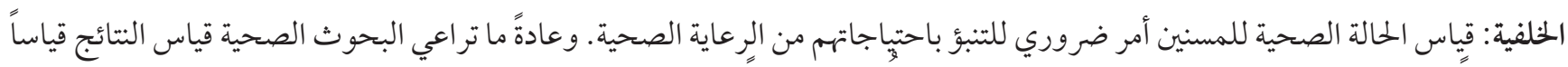

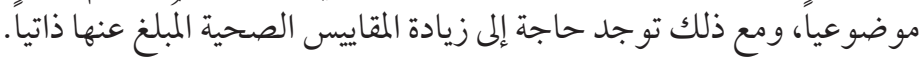

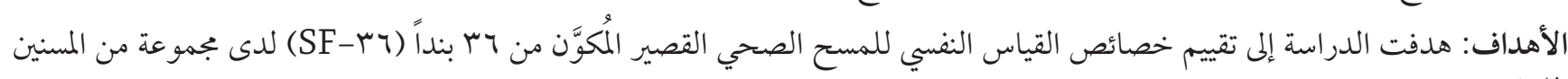

$$
\begin{aligned}
& \text { اللبنانين. }
\end{aligned}
$$

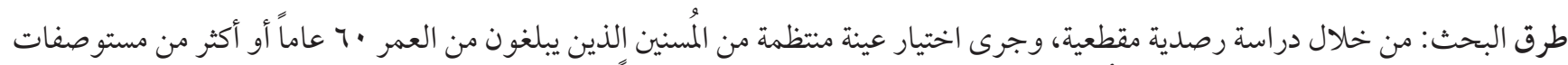

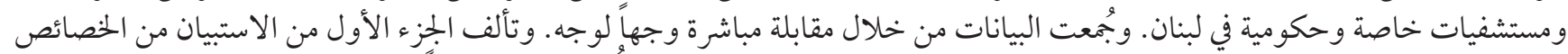

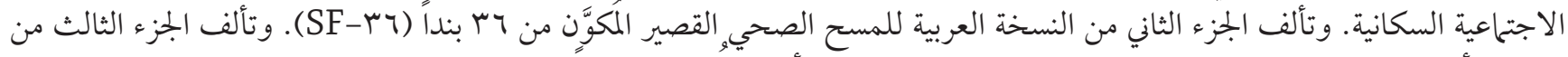

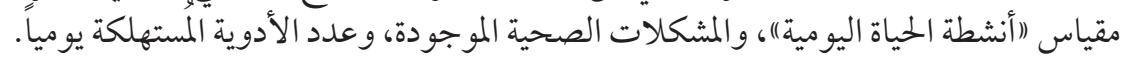

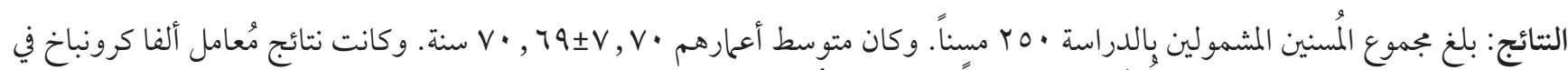

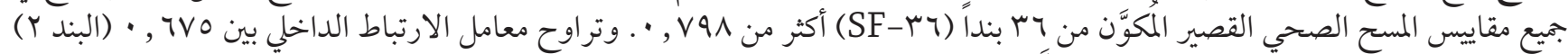

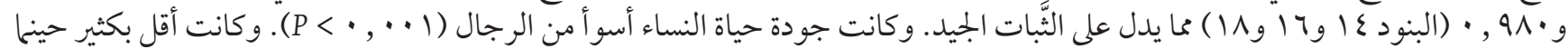

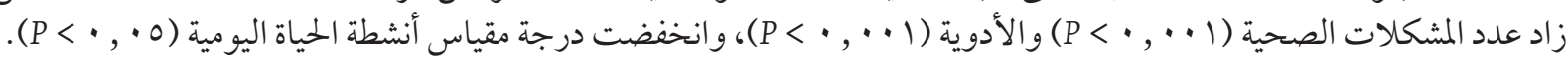

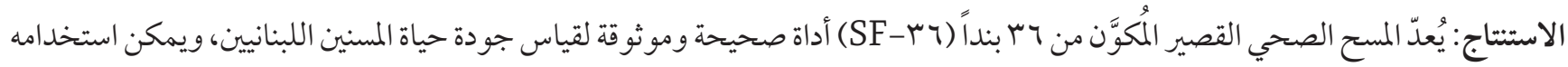

$$
\begin{aligned}
& \text { لرصد جودة حياة هذه الفئة السكانية. }
\end{aligned}
$$




\section{Références}

1. The World Oral Health Report 2003. Continuous improvement of oral health in the 21st century - the approach of the WHO Global Oral Health Programme. Geneva: World Health Organization, 2003 (WHO/NMH/NPH/ORH/03.2).

2. Costanza R, Fisher B, Ali S, Beer C, Bond L, Boumans R, et al. Quality of life: An approach integrating opportunities, human needs, and subjective well - being. Ecol Econ. 2007 Mar;61(2 - 3):267-76. https://doi.org/10.1016/j.ecolecon.2006.02.023

3. Ware JE Jr, Sherbourne CD. The MOS 36 - item short - form health survey (SF - 36). I. Conceptual framework and item selection. Med Care. 1992 Jun;30(6):473-83. https://doi.org/10.1097/00005650 - 199206000 - 00002 PMID:1593914

4. Berrío Valencia MI. Aging population: A challenge for public health. Colomb J Anesthesiol. 2012;40(3):192-94. https://doi. org/10.1016/j.rcae.2012.04.002

5. Osta NE, Hennequin M, Osta LE, Naaman NB, Geahchan N, Tubert - Jeannin S. État des lieux sanitaire et bucco - dentaire de la population gériatrique libanaise. East Mediterr Health J. 2015 Aug;21(5):349-53. PMID:26343124

6. Sabbah I, Drouby N, Sabbah S, Retel-Rude N, Mercier N. Quality of Life in rural and urban populations in Lebanon using SF - 36 Health Survey. Health Qual Life Outcomes. 2003 Aug 6;1:30. https://doi.org/10.1186/1477 - 7525 - 1 - 30 PMID:12952543

7. Sabbah I, Sabbah H, Khamis R, Sabbah S, Droubi N. Health related quality of life of university students in Lebanon: Lifestyles behaviors and socio - demographic predictors. Health. 2013;5(7):1-12. https://doi.org/10.4236/health.2013.57A4001

8. Beaton DE, Bombardier C, Guillemin F, Ferraz MB. Guidelines for the process of cross - cultural adaptation of self - report measures. Spine. 2000 Dec;25(24):3186-91. https://doi.org/10.1097/00007632 - 200012150 - 00014 PMID:11124735

9. Chahine LM, Bijlsma A, Hospers AP, Chemali Z. Dementia and depression among nursing home residents in Lebanon: a pilot study. Int J Geriatr Psychiatry. 2007 Apr;22(4):283-5. https://doi.org/10.1002/gps.1663 PMID:16977675

10. Nasser R, Doumit J. Validity and reliability of the Arabic version of Activities of Daily Living (ADL). BMC Geriatr. 2009 Mar;9:11. https://doi.org/10.1186/1471-2318-9-11 PMID:19327172

11. Nunnally JC, Bernstein IH. Psychometric theory. 3rd ed. New York: McGraw - Hill; 1994.

12. Institute of Medicine (US) Council on Health Care Technology; Mosteller F, Falotico - Taylor J, editors. Quality of Life and Technology Assessment: Monograph of the Council on Health Care Technology. Washington (DC): National Academies Press (US); 1989. http://www.ncbi.nlm.nih.gov/books/NBK235124/, consulté le 19 mars 2019) PMID:25144043

13. Terwee CB, Bot SD, de Boer MR, van der Windt DA, Knol DL, Dekker J, et al. Quality criteria were proposed for measurement properties of health status questionnaires. J Clin Epidemiol. 2007 Jan;60(1):34-42. https://doi.org/10.1016/j. jclinepi.2006.03.012 PMID:17161752

14. Butterworth P, Crosier T. The validity of the SF-36 in an Australian National Household Survey: demonstrating the applicability of the Household Income and Labour Dynamics in Australia (HILDA) Survey to examination of health inequalities. BMC Public Health. 2004 Oct;4:44. https://doi.org/10.1186/1471-2458 - 4 - 44 PMID:15469617

15. Tseng HM, Lu JF, Gandek B. Cultural issues in using the SF - 36. Health Survey in Asia: results from Taiwan. Health Qual Life Outcomes. 2003 Nov 26;1:72. https://doi.org/10.1186/1477- 7525 - 1 - 72 PMID:14641915

16. Leidy NK, Revicki DA, Genesté B. Recommendations for evaluating the validity of quality of life claims for labeling and promotion. Value Health. 1999 Mar-Apr;2(2):113-27. https://doi.org/10.1046/j.1524 - 4733.1999.02210.x PMID:16674343

17. Guyatt GH, Kirshner B, Jaeschke R. Measuring health status: what are the necessary measurement properties ? J Clin Epidemiol. 1992 Dec;45(12):1341-5. PMID:1460470

18. Khader S, Hourani MM, Al - Akour N. Normative data and psychometric properties of short form 36 health survey (SF - 36, version 1.0) in the population of north Jordan. East Mediterr Health J. 2011 May;17(5):368-74. PMID:21796947

19. Sullivan M, Karlsson J, Ware JE Jr. The Swedish SF - 36 Health Survey-I. Evaluation of data quality, scaling assumptions, reliability and construct validity across general populations in Sweden. Soc Sci Med. 1995 Nov;41(10):1349-58. https://doi. org/10.1016/0277-9536(95)00125 - Q PMID:8560302

20. Ware JE Jr. SF - 36 Health Survey Manual and Interpretation Guide. Boston, MA: The Health Institute, New England Medical Center; 1997.

21. Hopman WM, Towheed T, Anastassiades T, Tenenhouse A, Poliquin S, Berger C, et al. Canadian normative data for the SF - 36 health survey. Canadian Multicentre Osteoporosis Study Research Group. CMAJ. 2000 Aug 8;163(3):265-71. PMID:10951722

22. El Emrani L, Bendriss A, Senhaji M. Évaluation de la qualité de vie en relation avec la santé chez la population de Tétouan (Maroc) à l'aide du SF - 36: l'influence du niveau d'étude et de l'état matrimonial [Assessment of health - related quality of life in the population of Tetouan (Morocco) using the SF - 36: the influence of level of education and marital status]. Int J Innov Appl Stud. 2014 Aug;7(2) :815-22. 